Magdalena Skolimowska-Kulig

ORCID: 0000-0002-4748-7624

University of Wrocław

magdalena.skolimowska-kulig@uwr.edu.pl

\title{
Excess wealth transform for Pareto distributions
}

Date of submission: 1.01.2020; date of acceptance: 1.03.2020

JEL classification: C19, D31

Keywords: excess wealth transform, Pareto distributions

\section{Abstract \\ Excess wealth transform for Pareto distributions}

In the paper the notion of excess wealth transform and stochastic partial order based on it, introduced by Shaked and Shanthikumar (1998) are considered. The relations of the transform with the Lorenz curve and with certain variability measures are presented. The excess wealth transforms for Pareto type probability distributions are derived and their point-wise comparison is studied.

\section{Introduction}

The concept of variability is a basic one in statistics and related areas, such as reliability theory, economics, and actuarial science. Since common measures of variability, like variance, range, as well as coefficient of variation, are based on a single number and may occur to be insufficiently informative, other methods are considered in the literature. These methods involve probability distribution functions, their transforms and stochastic orders, which are important tools for measuring and comparing variabilities of probability distributions (or random variables). The well-known example of such a tool of great importance in assessing income and wealth inequality are the Lorenz curve and the Lorenz order (see for instance Marshall et al., 2010). The stochastic comparison of distributions has been a vital area of research in many diverse areas of statistics, probability and their applications in finance, actuarial and reliability theories, as well as economics. Many dif- 
ferent types of stochastic orders have been studied. The comprehensive discussion of them and their properties is available in Shaked and Shanthikumar (2007) and Muller and Stoyan (2002).

Shaked and Shanthikumar (1998) introduced what they call the excess wealth transform of the distribution function, which is intimately related to the unscaled Lorenz curve, and they defined the stochastic order based on point-wise comparison of the excess wealth transforms of two probability distributions. Independently at the same time the transform and the related order were studied, as the right spread transform and the right spread order, by Fernández-Ponce, Kochar and MuñozPerez (1998). The excess wealth order, its properties and relationships with other orders, as well as possible applications, were extensively studied by the aforementioned authors and others. The excess wealth order is considered mainly as a method of comparing risks in actuarial science (Denuit and Vermandele, 1999; Sordo, 2009; Belzunce et al., 2016) and a tool used in reliability theory (Kayid and Al-Dokar, 2009; Kochar et al., 2002). Also discussed are its applications in extreme risk analysis, auction theory (Kochar and $\mathrm{Xu}, 2013$ ) and analysis of packet transmission processes (Markovich and Krieger, 2013). Fernández-Ponce, Pellerey and Rodríguez-Griñolo (2011) defined and studied a multivariate generalisation of the excess wealth function and used it for a multivariate stochastic comparison.

In this paper attention is focused firstly on recalling the notion of excess wealth transforms and their relationships with basic statistical measures of income and wealth dispersion and inequality. In this part the representation of the Gini index via this transform is given. The second aim is to derive the excess wealth transforms for Pareto type probability distributions, which are viewed as being suitable for modelling income and wealth distributions, and to study the excess wealth order among these families of distributions.

\section{The excess wealth transform and the excess wealth order}

\subsection{Definition and basic properties}

Consider a random variable $\mathrm{X}$ with the probability distribution function $F(t)=P(X \leq t)$. The excess wealth transform of distribution F, introduced by Shaked and Shanthikumar (1998) and independently by Fernández-Ponce et al. (1998) as the right spread function, is defined as

$$
W_{F}(p)=\int_{F^{-1}(p)}^{\infty}[1-F(x)] d x, p \in[0,1] .
$$


In the above formula $F^{-1}$ is the inverse of $F$ (a quantile function), and in case the distribution $F$ is not strictly monotone, $F^{-1}(p)=\sup \{x: F(x) \leq p\}$.

In the context of economics $F$ if is considered as a distribution of wealth in some population, $F^{-1}(p)$ (a $p$ th quantile) is the is the largest wealth value such that $100 p \%$ of the population have at most that much and $\mathrm{W}_{\mathrm{F}}(p)$ may be thought of as the additional wealth (on top of the $p$ th quantile) of the richest $100(1-p) \%$ individuals in the population.

The transform (1) also written of the following form:

$$
W_{F}(p)=E\left[\left(X-F^{-1}(p)\right)^{+}\right],
$$

where $(X)^{+}=\max \{X, 0\}$. In actuarial literature the function (2) is called the expected shortfall at level $p$ and represents the expected shortfall of the portfolio with loss $X$ and solvency capital requirement $F^{-1}(p)$ (see Dhaene et al., 2006).

Usually the excess wealth transform and its properties are studied for nonnegative random variables, however the requirement that $X$ is a nonnegative random variable is not necessary for $W_{F}$ to be well defined, just the finite mean of $X$ is needed.

The excess wealth transform $W_{F}(p)$ is a decreasing function of $p, p \in[0,1]$. Notice that $W_{F}(1)=0$ and for nonnegative $X$ with distribution function $F$ and $F^{-1}(0)=0 W_{F}(0)=E(X)$. The excess wealth transform is location invariant and scale equivariant which means that for random variable $Y=a X+b$ for some real numbers $a>0$ and $b$, with the distribution function denoted by $G$, the excess wealth transform is of the form

$$
W_{G}(p)=a W_{F}(p), p \in[0,1],
$$

where $W_{F}$ is the excess transform of $X$.

The scale invariant version, called the scaled excess wealth transform (Kochar and $\mathrm{Xu}$ 2013) is defined as:

$$
S W_{F}(p)=\frac{W_{F}(p)}{E(X)}=\frac{\int_{F^{-1}(p)}^{\infty}[1-F(x)] d x}{\int_{0}^{\infty}[1-F(x)] d x}, \quad p \in[0,1],
$$

where the mean $E(X)$ is assumed to be finite and positive.

\section{Examples}

1) An equal (egalitarian) distribution, $X=c$ :

$$
W_{F}(p)=S W_{F}(p)=0, \quad p \in[0,1] .
$$

2) A uniform on $(a, b)$ :

$$
W_{F}(p)=\frac{b-a}{2}(1-p)^{2},
$$




$$
S W_{F}(p)=\frac{b-a}{b+a}(1-p)^{2}(\text { given } b \neq-a), \quad p \in[0,1 .
$$

3) An exponential with a mean $\lambda>0$ :

$$
\begin{gathered}
W_{F}(p)=\lambda(1-p), \\
S W_{F}(p)=1-p, \quad p \in[0,1] .
\end{gathered}
$$

Based on the point-wise comparison of excess wealth transforms a stochastic partial order is proposed (Shaked and Shanthikumar, 1998). It provides a natural way of comparing the riskiness or dispersion of two probability distributions. A random variable $X$ with distribution $F$ is said to be smaller than another random variable $Y$ with distribution $G$ in the excess wealth order (denoted by $X \leq_{e w} Y$ ) if

$$
W_{F}(p) \leq W_{G}(p) \text { for all } p \in[0,1] .
$$

It is worth mentioning that, in contrary to the Lorenz order, the excess wealth order is location invariant, which is directly implied by (3).

\subsection{The excess wealth transform and variability measures}

Suppose that $X$ is nonnegative with distribution function $F$ strictly increasing and $F^{-1}(0)=0$. Recall the definition of the Lorenz curve for nonnegative random variable $X$ with the distribution $F$ function and finite expected value $E(X)$ (Gastwirth 1972):

$$
L_{F}(p)=\frac{1}{E(X)} \int_{0}^{F^{-1}(p)} x d F(x), \quad p \in[0,1],
$$

where $F^{-1}$ is a quantile function given as $F^{-1}(p)=\sup \{x: F(x) \leq p\}$. If $F$ is a distribution of wealth (or income) in a given population, then $L_{F}(p)$ is the proportion of wealth of the poorest $p 100 \%$.

The equivalent to (5) representation of the Lorenz curve is given as:

$$
L_{F}(p)=\frac{1}{E(X)} \int_{0}^{p} F^{-1}(u) d u=\frac{\int_{0}^{p} F^{-1}(u) d u}{\int_{0}^{1} F^{-1}(u) d u}, \quad p \in[0,1] .
$$

Shaked and Shanthikumar (1998) revealed the relation of the excess wealth transform to the Lorenz curve:

$$
W_{F}(p)=E(X)\left[1+L_{F}(p)\right]-(1-p) F^{-1}(p), \quad p \in[0,1] .
$$

Variance and coefficient of variation may be expressed via the excess wealth transform and its scaled version (Fernández-Ponce et al., 1998): 


$$
\operatorname{Var}(X)=\int_{0}^{1}\left[\frac{W_{F}(p)}{1-p}\right]^{2} d p, \quad C V(X)=\left[\int_{0}^{1}\left[\frac{S W_{F}(p)}{1-p}\right]^{2} d p\right]^{1 / 2}
$$

as well as the truncated variance (Kochar and $\mathrm{Xu} 2013$ ):

$$
\operatorname{Var}\left(X \mid F^{-1}\left(p_{0}\right)\right)=\frac{1}{1-p_{0}} \int_{p_{0}}^{1}\left[\frac{W_{F}(p)}{1-p}\right]^{2} d p, 0 \leq p_{0}<1 .
$$

It also is possible to derive the Gini index from the excess wealth transform.

Proposition 1 For nonnegative random variable $X$ with distribution function $F$ strictly increasing the Gini index is of the form:

$$
\begin{gathered}
G(X)=\int_{0}^{1} S W_{F}(p) d p . \\
G(X)=2 \int_{0}^{1}\left(p-L_{F}(p)\right) d p=2 \int\left[p-\frac{1}{E(X)} \int_{0}^{p} F^{-1}(u) d u\right] d p \\
=1-\frac{2}{E(X)} \int_{0}^{1}(1-p) F^{-1}(p) d p .
\end{gathered}
$$

Notice that $W_{F}(p)=\int_{p}^{1} F^{-1}(u) d u-(1-p) F^{-1}(p)$. Hence

$$
\begin{aligned}
\int_{0}^{1} S W_{F}(p) d p= & \int_{0}^{1} \frac{W_{F}(p)}{E(X)} d p=\frac{1}{E(X)} \int_{0}^{1} p F^{-1}(p) d p-\frac{1}{E(X)} \int_{0}^{1}(1-p) F^{-1}(p) d p \\
& =\frac{1}{E(X)}\left(E(X)-\int_{0}^{1}(1-p) F^{-1}(p) d p\right)-\frac{1}{E(X)} \int_{0}^{1}(1-p) F^{-1}(p) d p=G(X)
\end{aligned}
$$

\section{Proof}

The Gini index is the double area between the Lorenz curve and the egalitarian line, so, using the representation (6) of the Lorenz curve it is expressed as:

\section{The excess wealth ordering of Pareto distributions}

Pareto distributions are useful modelling and predicting tools in a wide variety of socioeconomic contexts. One specific field of application is the size distribution of income. It was the context in which Vilfredo Pareto introduced the concept in his well-known economics text (Pareto 1897). Pareto observed that in many populations the number of individuals whose income exceeded a given level $x$ was well approximated by $C x^{\alpha}$ for some real $C$ and some $\alpha>0$. More precisely, such an approximation seems to be appropriate for large values of $x$ (which is 
called nowadays Paretian tail behaviour of income distribution). The parameter $\alpha$ depends on changes in the population and changes in the definition of income used in deriving the distribution.

As discussed in Arnold $(2008,2015)$ a lot of empirical studies and several stochastic models can be used to justify that in the upper tail, income distributions are reasonably well approximated by Pareto distribution. Since a variety of generalisations of this classical distribution have been proposed, including discrete versions and multivariate extensions, consequently, variants of the Pareto model are well accepted as default models for income and more general size distributions.

In the paper, four types of Pareto models are considered, according to Arnold's classification (Arnold, 2015). For each model the excess wealth transform as well as its scaled version are derived and the $\leq_{e w}$ order is studied.

\subsection{Pareto (I) distribution (the classical model)}

A random variable $X$ is said to have is said to have a Pareto (I), or classical Pareto distribution, if its distribution function is of the form:

$$
F(x ; \sigma, \alpha)=1-\left(\frac{x}{\sigma}\right)^{-\alpha}, x>\sigma,
$$

where $\sigma>0$ is a scale parameter and $\alpha$ is a positive slope parameter called is a positive slope parameter called Pareto's index (or an index of inequality).

If $\alpha>1$, the mean of $X$ exists, so does the excess wealth transform:

$$
\begin{gathered}
E(X)=\sigma \frac{\alpha}{\alpha-1}, \\
W_{F}(p)=\frac{\sigma}{\alpha-1}(1-p)^{\frac{\alpha-1}{\alpha}}, p \in[0,1], \\
S W_{F}(p ; \sigma, \alpha)=\frac{1}{\alpha}(1-p)^{\frac{\alpha-1}{\alpha}}, p \in[0,1] .
\end{gathered}
$$

The point-wise comparison of the excess wealth transforms according to the scale parameter is trivial bearing in mind (3). Moreover, the arrangement of slope parameters implies the $\leq_{e w}$ order among Pareto (I) distributions.

Proposition 2 Let $X_{1}$ and $X_{2}$ be the Pareto (I) random variables with indexes $\left(\sigma_{1}, \alpha_{1}\right)$ and $\left(\sigma_{2}, \alpha_{2}\right)$ respectively, and $\sigma_{1} \leq \sigma_{2}$. If $1<\alpha_{2} \leq \alpha_{1}$ then $X_{1} \leq_{\mathrm{ew}} X_{2}$.

Remark Notice that for Pareto (I) distributions with the same scale parameter the reverse implication holds. Thus for $X_{1}$ and $X_{2}$ with indexes $\left(\sigma, \alpha_{1}\right)$ and $\left(\sigma, \alpha_{2}\right)$ respectively, $\alpha_{1}, \alpha_{2}>1$, the relation $X_{1} \leq_{\mathrm{ew}} X_{2}$ is equivalent to $\alpha_{2} \leq \alpha_{1}$. Moreover, given that the Gini index is of the form $G(X)=\frac{1}{2 \alpha-1}$, the $\leq_{e w}$ ordering within the Pareto (I) family may be defined by the relationship among Gini indexes. 


\subsection{Pareto (II)}

The introduction of a location parameter $\mu$ to the previous model leads to the second type of Pareto distribution with the distribution function

$$
F(x ; \mu, \sigma, \alpha)=1-\left[1+\frac{x-\mu}{\sigma}\right]^{-\alpha}, x>\mu, \sigma, \alpha>0 .
$$

In this model

$$
F^{-1}(p ; \mu, \sigma, \alpha)=\sigma\left[(1-p)^{-\frac{1}{\alpha}}-1\right]+\mu
$$

and for $\alpha>1$ :

$$
\begin{gathered}
E(X)=\frac{\sigma}{\alpha-1}+\mu \\
W_{F}(p)=\frac{\sigma}{\alpha-1}(1-p)^{\frac{\alpha-1}{\alpha}}, \quad p \in[0,1] \\
S W_{F}(p)=\frac{\sigma}{\mu(\alpha-1)+\sigma}(1-p)^{\frac{\alpha-1}{\alpha}}, \quad p \in[0,1]
\end{gathered}
$$

The function $W_{F}$ is of the same form as for the Pareto (I) model. Therefore, the same conditions imply $\leq_{e w}$ ordering.

\subsection{Pareto (III)}

A Pareto (III) distribution is defined by the distribution function

$$
F(x ; \mu, \sigma, \gamma)=1-\left[1+\left(\frac{x-\mu}{\sigma}\right)^{\frac{1}{\gamma}}\right]^{-1}, x>\mu, \quad \sigma, \gamma>0
$$

A parameter $\gamma$ is called an index of inequality, moreover for $\mu=0$ it is exactly equal to the Gini index. In the considered model

$$
F^{-1}(p)=\sigma\left[(1-p)^{-1}-1\right]^{\gamma}+\mu,
$$

and for $0<\gamma<1$ the mean of $X$ exists and can be expressed as

$$
E(X)=\Gamma(1+\gamma) \Gamma(1-\gamma)+\mu,
$$

where $\Gamma$ is the gamma function. Then

$$
\begin{gathered}
W_{F}(p)=\sigma \gamma \mathrm{B}(1-p ; 1-\gamma, \gamma), p \in[0,1], \\
S W_{F}(p)=\frac{\sigma}{\sigma \mathrm{B}(1-\gamma, \gamma)+\mu / \gamma} \mathrm{B}(1-p ; 1-\gamma, \gamma), \quad p \in[0,1],
\end{gathered}
$$

where $\mathrm{B}(a, b)$ and $\mathrm{B}(x ; a, b)$ are beta and incomplete beta functions, respectively. 
If $\mu=0$ the scaled excess transform can be expressed as a regularised version of incomplete beta function:

$$
S W_{F}(p)=\mathrm{I}(1-p ; 1-\gamma, \gamma), \quad p \in[0,1] .
$$

Proposition 3 Let $X_{1}$ and $X_{2}$ be the Pareto (III) random variables with indexes $\left(\sigma_{1}, \mu_{1}, \gamma_{1}\right)$ and $\left(\sigma_{2}, \mu_{2}, \gamma_{2}\right)$ respectively, and $\sigma_{1} \leq \sigma_{2}$. If then $X_{1} \leq_{e w} X_{2}$.

Proof Notice that

$$
W_{F_{2}}(p)-W_{F_{1}}(p)=\left(\sigma_{2}-\sigma_{1}\right) \int_{0}^{1-p}\left[\gamma_{2} x^{-\gamma_{2}}(1-x)^{\gamma_{2}-1}-\gamma_{1} x^{-\gamma_{1}}(1-x)^{\gamma_{1}-1}\right] d x
$$

and the integrand changes its sign just once, from + to - . Therefore, to prove $W_{F_{2}}(p)-W_{F_{1}}(p) \geq 0$ for all $p \in[0,1]$ it is sufficient to show that the inequality holds for $p=0$. In the Pareto (III) model $W_{F}(0)=\sigma \gamma B(1-\gamma, \gamma)$. Furthermore, the beta function satisfies the identity $B(1-x, x)=\frac{\pi}{\sin (\pi x)}$, so

$W_{F}(0)=\sigma \frac{\pi \gamma}{\sin (\pi \gamma)}$. Since the last expression is an increasing function of $\gamma$, $0<\gamma<1$, and $\sigma_{2} \geq \sigma_{1}$, the inequality $W_{F_{2}}(0)-W_{F_{1}}(0) \geq 0$ is satisfied $\bullet$.

As for the Pareto (I) and (II) models, also in this case for given scale parameter the $\leq_{e w}$ ordering of distributions corresponds to ordering of parameters of inequality.

\subsection{Pareto (IV)}

The more general Pareto model is given by the distribution function

$$
F(x ; \mu, \sigma, \gamma, \alpha)=1-\left[1+\left(\frac{x-\mu}{\sigma}\right)^{\frac{1}{\gamma}}\right]^{-\alpha}, x>\mu, \quad \sigma, \alpha, \gamma>0 .
$$

In this model

$$
F^{-1}(p)=\sigma\left[(1-p)^{-1 / \alpha}-1\right]^{\gamma}+\mu .
$$

For $\alpha>1$ and $0<\gamma<1$ :

$$
\begin{gathered}
E(X)=\sigma \frac{\Gamma(1+\gamma) \Gamma(\alpha-\gamma)}{\Gamma(\alpha)}+\mu, \\
W_{F}(p)=\sigma \gamma \mathrm{B}\left((1-p)^{1 / \alpha} ; \alpha-\gamma, \gamma\right), \\
S W_{F}(p)=\frac{\sigma}{\sigma \mathrm{B}(\alpha-\gamma, \gamma)+\mu / \gamma} \mathrm{B}\left((1-p)^{1 / \alpha} ; \alpha-\gamma, \gamma\right),
\end{gathered}
$$

and if $\mu=0$, the scaled version is

$$
S W_{F}(p)=\mathrm{I}\left((1-p)^{1 / \alpha} ; \alpha-\gamma, \gamma\right) .
$$


The Pareto (IV) family is neither $\leq_{e w}$ ordered according to $\alpha$ nor $\gamma$. To verify it the following examples may be considered.

\section{Examples}

1) Let $X_{1}$ be Pareto (IV) with parameters $\sigma=1, \mu=0, \gamma=0.5$ and $\alpha_{1}=1.5$, while $X_{2}$ is Pareto (IV) with parameters $\sigma=1, \mu=0, \gamma=0.5$ and $\alpha_{2}=2.5$. It is quite straightforward to find $W_{F_{1}}$ and $W_{F_{2}}$ and to verify that neither $X_{1} \leq{ }_{e w} X_{2}$ nor $X_{2} \leq{ }_{e w} X_{1}$.

2) Let $X_{1}$ be Pareto (IV) with parameters $\sigma=1, \mu=0, \gamma_{1}=0.2$ and $\alpha=10$, while $X_{2}$ is Pareto (IV) with parameters $\sigma=1, \mu=0, \gamma_{2}=0.6$ and $\alpha=10$. Since for instance $W_{F_{1}}(0.09)>W_{F_{2}}(0.09)$ and $W_{F_{1}}(0.7)<W_{F_{2}}(0.7)$ neither $X_{1} \leq_{e w} X_{2}$ nor $X_{2} \leq_{e w} X_{1}$.

\section{Conclusions}

In the paper the definition of the excess wealth transform and its properties in the context of measuring and comparing income distributions are recalled. It is shown that for Pareto (I), (II) and (III) models the excess wealth order is implied by a certain arrangement of their parameters. In particular, comparing distributions with regard to their excess wealth transforms within the aforementioned families with the same scale parameter is equivalent to a comparison of their inequality parameters, and in case the parameter of location is zero (Pareto (II) and (III)) it coincides with ordering their Gini indexes.

\section{References}

Arnold, B.C. (2008). Pareto and Generalized Pareto Distributions. In D. Chotikapanich (ed.) Modeling Income Distributions and Lorenz Curves. Economic Studies in Equality, Social Exclusion and Well-Being, 5. New York, NY: Springer, 119-145. DOI:10.1007/978-0-387-72796-7_7.

Arnold, B.C. (2015). Pareto Distributions. Boca Raton, FL: CRC Press.

Belzunce, F., Martínez-Riquelme, C., Ruiz, J.M., Sordo, M.A. (2016). On sufficient conditions for the comparisons in the excess wealth order and spacings. Journal of Applied Probability, 53, 33-46. DOI:10.1017/jpr.2015.6.

Denuit, M., Vermandele, C. (1999). Lorenz and excess wealth orders, with applications in reinsurance theory. Scandinavian Actuarial Journal, 2,170-185. DOI:10.1080/03461239950132642.

Dhaene, J., Vanduffel, S., Goovaerts, M.J., Kaas, R., Tang, Q., Vyncke, D. (2006). Risk measures and comonotonicity:Areview. Stochastic Models, 22, 573-606.DOI:10.1080/15326340600878016.

Fernández-Ponce, J.M., Pellerey, F., Rodríguez-Griñolo, M.R. (2011). A characterization of the multivariate excess wealth ordering. Insurance: Mathematics and Economics, 49, 410-417. DOI:10.1016/j.insmatheco.2011.07.001.

Fernández-Ponce, J.M., Kochar, S.C., Muñoz-Perez, J. (1998). Partial Orderings of Distributions Based on Right-Spread Functions. Journal of Applied Probability, 35(1), 221-228. DOI:10.1239/jap/1032192565.

Gastwirth, J. (1972). The Estimation of the Lorenz Curve and Gini Index. The Review of Economics and Statistics, 54 (3), 30-316. DOI:10.2307/1937992.

Ekonomia - Wroclaw Economic Review 26/, 2020

(C) for this edition by CNS 
Kayid, M., Al-Dokar, S. (2009). Some Results on the Excess Wealth Order with Applications in Reliability Theory. Contemporary Engineering Sciences, 2, 269-282.

Kochar, S., Li, X., Shaked, M. (2002). The total time on test transform and the excess wealth stochastic orders of distributions. Advances in Applied Probability, 34(4), 826-846.

Kochar, S., Xu, M. (2013). Excess Wealth Transform with Applications. In H. Li, X. Li (eds.), Stochastic Orders in Reliability and Risk. Lecture Notes in Statistics, 208, 273-288. New York, NY: Springer.

Markovich, N.M., Krieger, U.R. (2013). Analysis of Packet Transmission Processes in Peer-to-Peer Networks by Statistical Inference Methods. In E. Biersack, C. Callegari, M. Matijasevic (eds.), Data Traffic Monitoring and Analysis. Lecture Notes in Computer Science, 7754, 104-119. Berlin: Springer. DOI:10.1007/978-3-642-36784-7_5.

Marshall, A.W., Olkin, I., Arnold, B.C. (2010). Stochastic Ordering. In Inequalities: Theory of Majorization and Its Applications. Springer Series in Statistics, 693-756. New York, NY: Springer.

Muller, A., Stoyan, D. (2002). Comparison Methods for Stochastic Models and Risks. Chichester: Wiley.

Pareto, V. (1897). Cours d'Economie Politique, 2. Lausanne.

Shaked, M., Shanthikumar, J.G. (1998). Two probability orders. Probability in Engineering and Informational Sciences, 12, 1-23. DOI:10.1017/S0269964800005039.

Shaked, M., Shanthikumar, J.G. (2007). Stochastic Orders. New York: Springer.

Sordo, M.A. (2009). Comparing tail variabilities of risks by means of the excess wealth order. Insurance: Mathematics and Economics, 45, 466-469.

Ekonomia - Wroclaw Economic Review 26/, 2020

(C) for this edition by CNS 